\title{
Estudio epidemiológico de las parasitosis intestinal y su influencia en el estado nutricional de los niños de Potao - Barranca - 2010
}

\author{
Epidemiology study of intestinal parasites and their influence on the nutritional status of \\ children Potao - Barranca - 2010 \\ ${ }^{1}$ Juan Díaz O. a , ${ }^{1}$ Eladio Sánchez C. ${ }^{\text {, }},{ }^{1}$ José Polo C. ${ }^{\text {c }}{ }^{2}$ Segundo Ponte V. a
}

\section{RESUMEN}

Siendo la parasitosis intestinal un problema de salud pública en el Perú, la investigación tuvo como objetivo describir las características parasitológicas y epidemiológicas sobre la parasitosis intestinal y su efecto en el estado nutricional de los niños de Potao - Barranca. Se realizó un estudio descriptivo correlacional, de corte transversal. El universo estuvo constituido por 290 niños menores hasta los 14 años de edad de la comunidad de Potao - Barranca, de la cual se obtuvo una muestra aleatoria simple de 71 niños, y se utilizó un cuestionario para encuestar a los padres de familia, el que estuvo organizado con preguntas cerradas, dicotómicas y de opciones múltiples que abarcara datos relacionados con aspectos epidemiológicos asociados a las infecciones parasitarias. Se hicieron pruebas parasitológicas para determinar la prevalencia y los tipos de parásitos presentes. Asimismo se realizó antropometría (peso-talla) a todos los niños.

Los datos fueron procesados en el Programa computarizado SPSS versión 15.0. Se hizo un análisis univariado y bivariado.

Se encontró una prevalencia de parasitosis en la población infantil menor de 14 años de $71,8 \%$. El $58.8 \%$ de los niños presentan monoparasitosis y el $41.2 \%$ poliparasitosis. Los parásitos encontrados con mayor frecuencia fueron: Giardia lamblia, seguida de Enterobius vermicularis y la bacteria Escherichia coli. La presencia de parasitosis no tuvo relación significativa con el estado nutricional de los niños estudiados, aunque se reportó desnutrición crónica en un $17 \%$ en los menores de 5 años y $16.7 \%$ entre 5 a 14 años; La desnutrición aguda en un $4.3 \%$ en los menores de 5 años. Se encontró que el piso de tierra, la mala eliminación de excretas y la presencia de animales en casa fueron significativos para la presencia de parasitosis en los niños. Fstas características indican la falta de saneamiento ambiental que puede estar relacionado con la presencia de parasitosis.

Palabras claves: Salud pública, infecciones parasitarias, pruebas parasitológicas, epidemiología.

\begin{abstract}
Intestinal parasites being a public health problem in Peru, the study aimed to describe the characteristics and epidemiological parasitoscopic intestinal parasites and their effect on nutritional status of children of Potao Barranca. We performed a correlational, transversal descriptive study. 'The universe had 290 children under 14 years of community Potao - Barranca. The sample was obtained from children whose parents attended the lecture on parasites, which was obtained in 71 cases. A questionnaire was used to survey parents, which was organized with closed questions, dichotomous, multiple choice covering epidemiological data related to parasitic infections. Parasitoscopic tests were to determine the prevalence and types of parasites. It was also made anthropometry (weight - size) to all children.

The data were processed by SPSS version 15.0 computer program. There was a univariate and bivariate analysis. The prevalence of parasitic infections in children fewer than 14 years was $71.8 \%$. The $58.8 \%$ of children have monoparasitosis and $41.2 \%$ poly-parasitic infection. The most common parasites found were Giardia lamblia, Enterobius vermicularis followed and Entamoeba coli. The presence of parasites was not associated with nutritional status of children studied; however chronic malnutrition was reported by $17 \%$ in children fewer than 5 years and $16 \%$ between 5 and 14 years old, acute malnutrition at $4.3 \%$ in fewer than 5 years. It was found that the absence of floor, poor disposal of excreta and the presence of pets at home were associated for the presence of parasites in children. These data indicate the bad sanitary health habits can be related to the presence of parasites.
\end{abstract}

Keywords: public health, parasitic infections, parasitological tests, epidemiology.

\footnotetext{
Universidad Nacional de Jaén. Cajamarca.

${ }^{2}$ Universidad Nacional de Barranca.

${ }^{\mathrm{a}}$ Licenciado en Estadística, ${ }^{\mathrm{b}}$ Licenciado en Matemática, ${ }^{\mathrm{C}}$ Biologo.
} 


\section{INTRODUCCIÓN}

En los últimos años se ha descrito mucho acerca de las enfermedades parasitarias y su relación con el estado nutricional. Sin embargo para miles de niños en el mundo aún persisten los efectos de la desnutrición y la parasitosis, relacionados con aspectos epidemiológicos por la agresión de la enfermedad, ocasionando anualmente el incremento de casos de parasitosis. Dicha afección es el tema principal de investigación (Booker et al., 1999). Según (Arancón, 1990) las características epidemiológicas, los casos de parasitosis y el desequilibrio del estado nutricional reflejan la estrecha relación entre el estado nutricional y la parasitosis creando un problema en la salud pública por lo que se espera su mejoría y la importancia práctica en un futuro próximo.

La investigación se realizó en un grupo de personas hasta los 14 años de edad, de los cuales se deduce la situación nutricional parasitaria de la población en estudio. En el presente documento se define la problemática, así como su magnitud, trascendencia y vulnerabilidad de la relación de parasitosis; por lo cual el objetivo del presente trabajo es determinar las características epidemiológicas y epidemiológicas de la parasitosis intestinal y su influencia en estado de salud nutricional de la comunidad de Potao - Barranca. Debemos considerar los antecedentes de la epidemiología y significación pública de las enfermedades parasitarias, relacionado con la etiología del estado nutricional. Este trabajo de investigación contiene la hipótesis (Ho) que es una propuesta tentativa de la problemática planteada, aclarando así la operacionalización, determinación de la muestra, métodos y procedimientos.

\section{MATERIAL YMÉTODOS}

\section{Tipo de Investigación.}

Descriptivo, porque va a describir las características epidemiológicas y los tipos de parásitos más frecuentes que se observaron en la población infantil; de igual manera el esludio corresponde a una investigación correlacional, al asociar la parasitosis intestinal y su efecto en el estado nutricional de la población infantil.

\section{Diseño de la Investigación.}

El trabajo de investigación se inscribió en el diseño de investigación no experimental y se utilizó los diseños transversales, descriptivos y correlaciónales.

\section{Fuente y Técnica de Recolección de Datos.} La fuente de datos fue primaria porque las muestras para la evaluación parasitológica y nutricional se tomó directamente de cada niño. La técnica empleada para la recolección de datos fue la encuesta a través de un cuestionario.

El examen parasitológico se realizó en forma seriada y el examen microscópico se hizo en forma directa previamente realizando el Método de Concentración de Berman Modificado en copa y para el diagnóstico de Enterobius vermicularis se realiza el Método de Grahann; y para identificar el resto de parásitos se hizo un examen directo de heces (Botero y Restrepo 1998).

Para la evaluación nutricional se tomó el peso y talla de los niños y niñas que estuvieron en la investigación, los cuales se confrontaron con su edad, y se utilizó los parámetros del Ministerio de Salud del Perú (INEI, 2011).

\section{Población o Universo.}

Estuvo representada por todos los niños hasta los 14 años $(\mathrm{N}=290)$ que viven en la comunidad de Potao del Distrito de Barranca durante los meses de Febrero -Agosto 2010.

\section{Unidad de Análisis y Muestra.}

- Unidad de análisis: La unidad de análisis estuvo conformada por un niño con una edad menor o igual a los 14 años que vive en la Comunidad de Potao.

- Muestra:El tamaño de la muestra se determinó mediante la siguiente fórmula (Martínez, 2011):

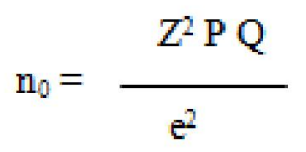

Dónde:

$$
\begin{aligned}
1-\alpha & =\text { Confianza }=95 \% \\
\mathrm{Z}^{2} & =\text { Coeficiente de confianza }=1.96 \\
\mathrm{P} & =\text { Proporción esperada de niños con } \\
& \text { parasitosis: } 0.935 \\
\mathrm{Q} & =1-\mathrm{P}=0.065 \\
\mathrm{P} & =\text { Precisión }=0.05 \\
\mathrm{~N} & =\text { Tamaño de la población: } 290
\end{aligned}
$$

$$
\mathrm{m}_{0}=\frac{\mathrm{Z}^{2} \mathrm{PQ}}{\mathrm{e}^{2}}=\frac{1.96^{2}(0.935)(0.065)}{0.05^{2}}=93.389
$$

Muestra ajustada

$$
\mathrm{n}=\frac{\mathrm{n}_{4}}{1+\mathrm{n}_{0} / \mathrm{N}}=\frac{93.389}{1+93.389 / 290}=71
$$

La muestra estuvo constituida por 71 niños menores de 14 años de edad que viven en la comunidad de Potao, y cumplieron con los criterios de inclusión y exclusión. 
Criterios de Inclusión (Batista y col., 1992).

Niños que tuvieron el consentimiento informados de los padres.

Niños menores de 14 años.

Niños que no estuvieron recibiendo tratamiento para parasitosis.

\section{Criterios de Exclusión (Batista y col., 1992).}

Todos aquellos que no dieron el consentimiento informado.

Niños que estuvieron recibiendo tratamiento para parasitosis.

Personas mayores de 14 años.

\section{Análisis Estadístico o Interpretación de la Información.}

El análisis estadístico fue computarizado empleando el Paquete Estadístico SPSS Versión 15. El plan de análisis consistió en el análisis univariado calculando frecuencias y porcentajes para las variables categóricas. El análisis bivariado consistió en buscar asociaciones entre los tipos de parásitos y las características epidemiológicas, la asociación estadística de las variables categóricas se realizó con el test de Chi-cuadrado. El valor de $p<0.05$ fue considerado como nivel crítico de significación.

La tabulación de datos se realizó a través de una serie de cuadros que permitieron la presentación de la información en forma ordenada.

\section{Ética de la Investigación.}

Para el estudio se tomó el consentimiento previo de los encuestados, así como del apoderado (a) del niño (a) para poder tomar las muestras, por lo cual se requirió del consentimiento informado.

\section{RESULTADOS}

\section{Resultados descriptivos.}

Respecto a las características epidemiológicas, tenemos en primer lugar que los niños a los que se les tomaron las pruebas para determinar la presencia de parasitosis fueron 71 , de los cuales el 71,8 \% (51 niños) tuvieron presencia de parásitos y el $28,2 \%$ (20 niños) no lo presentaron (Figura 1).

Teniendo en cuenta el porcentaje de niños parasitados, en la distribución por edades se tuvo que los menores de un año fueron el 19,6\% (10 niños), de un año el 11,8 \% (6 niños), de dos años el $13,7 \%$ (7 niños), de tres a cuatro años $15,7 \%$ (8 niños), de cinco a nueve años el $19,6 \%$ (10 niños) y de 10 a 14 años el 19,6\%(10 niños) (Figura 2).

Respecto a los niños parasitados y las características de la vivienda, tenemos que se encontró hacinamiento en un $39.2 \%$ de las familias. E1 material que predominó en las paredes de las viviendas fueron materiales precarios en un $82,4 \%$, en el techo los materiales precarios fue de 78,4 \% y en el piso el $84,3 \%$ de tierra, este último fue altamente significativo $\left(\mathrm{X}^{2}=20.449, \mathrm{p}<0.05\right)$. El $62.7 \%$ tiene abastecimiento de agua dentro de la vivienda. Respecto al tipo de servicio higiénico el $90,2 \%$ de las familias utilizó pozo ciego, acequia o no tiene el servicio, esto fue altamente significativo $\left(\mathrm{X}^{2}=14.341, \mathrm{p}<0.05\right)$.

Respecto al número de especies parasitarias por hospedador se encontró que el $58.8 \%$ presento un tipo de parásito y el $41.2 \%$ presento varios tipos de parásitos. (Figura 3).

En los niños de $0-4$ años, el parásito que más prevaleció fue Giardia lamblia con un $66 \%$, seguida por Enterobius vermicularis en $27.7 \%$, Entamoeba coli con $14.9 \%$, Hymenolepis nana y Ascaris lumbricoides representada con un $10.6 \%$ cada uno. En los niños de 5 a 14 años el parásito más frecuente que se encontró fue Giardia lamblia en un $83.3 \%$, seguida por Enterobius vermicularis en $41.7 \%$, Entamoeba coli con $25.0 \%$, Hymenolepis nana con $20.8 \%$ y Ascaris lumbricoides con un $12.5 \%$ (Figura 4 ).

Respecto al estado nutricional en los niños de 0 - 4 años: el $63.8 \%$ no presentó malnutrición, el $17 \%$ presentó desnutrición crónica, el $12.8 \%$ sobrepeso, el $4.3 \%$ desnutrición aguda, y el $2.1 \%$ riesgo nutricional. El estado nutricional de los niños de 514 años: el $54.2 \%$ no presentó malnutrición, el 16.7 $\%$ tuvo desnutrición crónica, el $12.5 \%$ riesgo de delgadez, el $8.3 \%$ sobrepeso y el $8.3 \%$ tiene obesidad. (Figura 5).

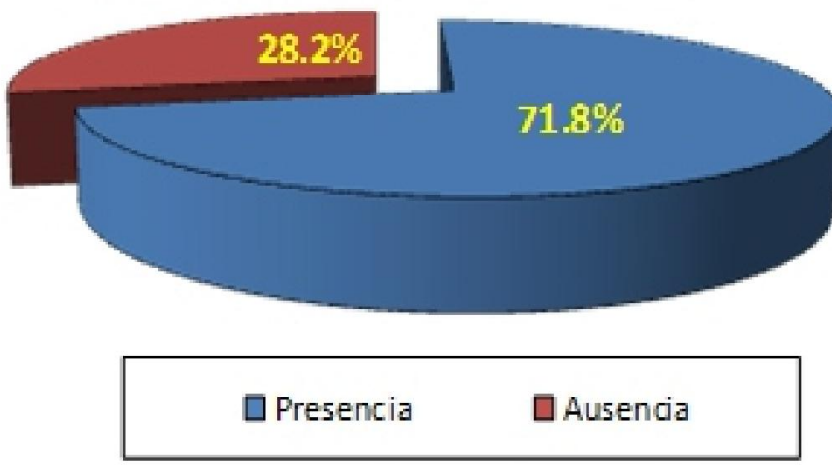

Figura 1: Distribución de los niños estudiados según presencia de parásitos Potao- 2010.

Fuente: Encuesta Epidemiológica 


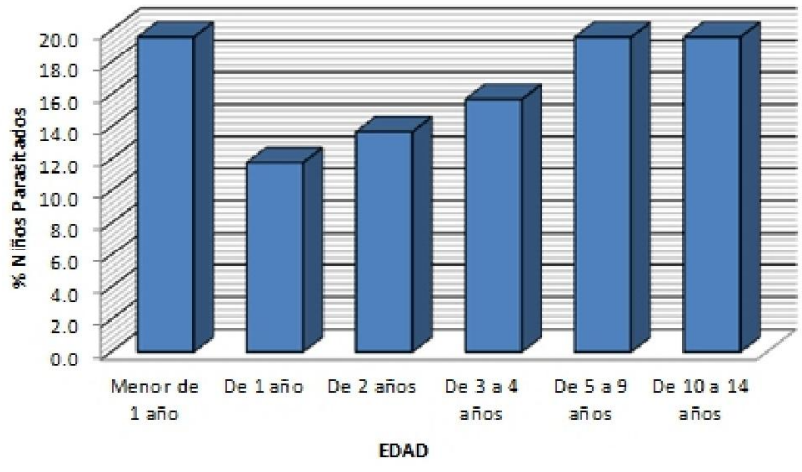

Figura 2: Distribución de niños parasitados según edad. Potao - 2010.

Fuente: Encuesta Epidemiológica.

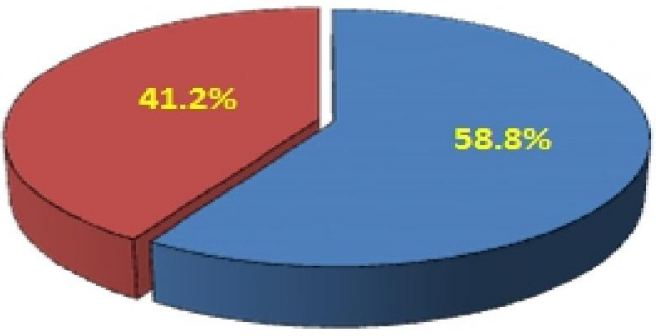

口Monoparasitos aMultiparas itos

Figura 3: Número de especies parasitarias por hospedador. Potao - 2010.

Fuente: Encuesta Epidemiológica.

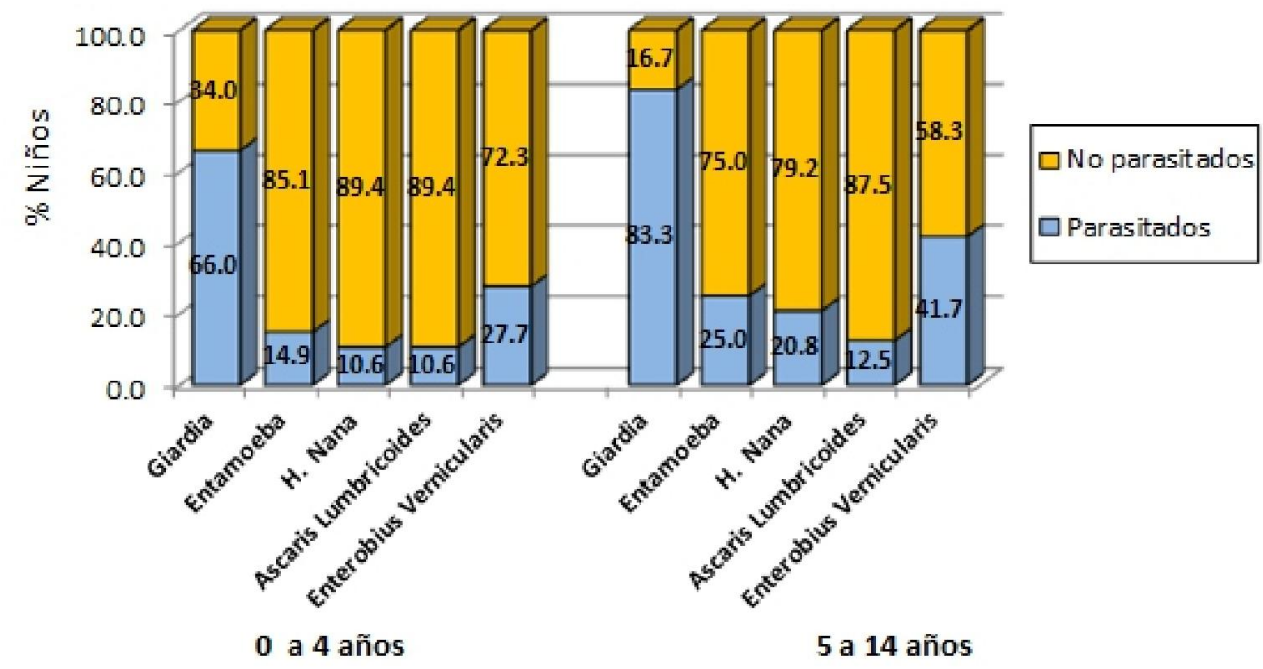

Figura 4: Prevalencia de parásitos intestinales en niños. Potao-2010.

Fuente: Encuesta Epidemiológica.

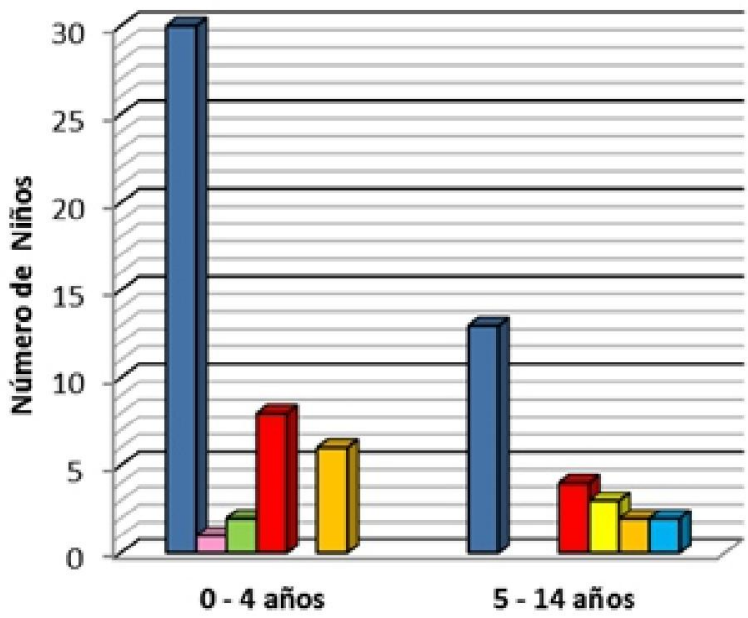

$\square$ Normal

$\square$ Riesgo Nutricional

口Desnutrición Aguda

口Desnutrición Crónica

口Riesgo de delgadez

$\square$ Sobrepeso

口Obesidad

Figura 5: Estado nutricional en niños según grupos de edad. Fuente: Encuesta Epidemiológica. 


\section{Resultados para la contrastación de la hipótesis}

\section{Hipótesis}

Ho: La presencia de parasitosis intestinal en los niños de Potao - Barranca, no influye significativamente en su estado nutricional.

H1: La presencia de parasitosis intestinal en los niños de Potao - Barranca, influye significativamente en su estado nutricional.

$$
\alpha=0.05
$$

\section{Estadístico de prueba}

$$
\begin{gathered}
\chi^{2}=\sum_{i=1}^{r} \sum_{j=1}^{k} \frac{\left(o_{i j}-e_{i j}\right)^{2}}{e_{i j}}=1.989 \\
\mathrm{P}=0.158
\end{gathered}
$$

\section{Decisión}

Dado que $\mathrm{P}=0.158>0.05$, entonces no se debe rechazar Ho.

\section{Conclusión}

Podemos afirmar con un $95 \%$ de confianza que la presencia de parasitosis intestinal, no influye significativamente en el estado nutricional de los niños de Potao de la Provincia de Barranca.

\section{DISCUSIÓN}

Fn el Perú no se tienen cifras precisas de prevalencia de parasitosis intestinal a nivel nacional, pero se puede afirmar que la prevalencia es alta ya que diversos estudios realizados en departamentos de la sierra y selva peruana muestran prevalencias mayores del $95 \%$, mientras que la prevalencia de enteroparásitos patógenos varía entre $62.3 \%$ y 64 $\%$. Se menciona que uno de cada tres peruanos porta uno o más parásitos en el intestino. La distribución de la parasitosis intestinal se presenta según las regiones geográficas del país (costa, sierra y selva). En este trabajo observamos que la población infantil menor o igual a los 14 años presentó parasitosis en un $71,8 \%$, este resultado se compara con el de Mendoza D. y col. 2006, que encontraron un $89 \%$ de resultados positivos a parasitosis y Raymundo M. y col. 2002. Quienes encontraron que la prevalencia de parasitosis intestinal fue alta, el 100 $\%$ de ellos tenían parásitos o comensales y el $64 \%$ alojaban patógenos con lo que se demuestra que la prevalencia es alta, por lo que deberíamos hacer un seguimiento oportuno e inmediato por el alto número de niños parasitados.

Teniendo en cuenta el porcentaje de niños parasitados, en la distribución por edades, se tuvo que las edades fluctuaron entre 0 a 14 años. En el estudio de Mendoza D. y col. 2006, las edades fluctuaron entre los 6 y 13 años.

El material que predominó en las paredes de las viviendas fueron materiales precarios en un $82,4 \%$, en el techo los materiales precarios fueron de 78,4\% $y$ en el piso el $84,3 \%$ fue de tierra, este último fue altamente significativo $\left(\mathrm{X}^{2}=20.449, \mathrm{p}<0.05\right)$, al igual que otras investigaciones donde se encontró una asociación significativa; como se sabe el contagio de los parásitos intestinales se establece en niños por contacto con la tierra, debido a que en ella y a través de la materia fecal se depositan los huevos de parásitos. Pasado unos días y si la temperatura y la humedad son adecuadas, los huevos se transforman en larvas que son las infectantes.

Respecto al tipo de servicio higiénico, el 90,2\% de las familias usa pozo ciego, acequia o no tiene el servicio, esto fue altamente significativo $\left(\mathrm{X}^{2}=\right.$ 14.341, $\mathrm{p}<0.05$ ) para la presencia de parasitosis. Esto es muy similar al estudio de Díaz et al. 2002. Quienes encontraron que el $100 \%$ de las viviendas no estaban conectadas a la red de cloacas. La eliminación final de las excretas se realizaba principalmente en fosas rudimentarias $(54.9 \%$ ) y en pozos sépticos $(43.7 \%)$. Si se dejan las excretas al aire libre, sus parásitos pueden contaminar el agua, el mar, los ríos, los suelos de cultivos. La importancia del sistemas de alcantarillado radica en que disminuye o anula la mala eliminación de las excretas que puede contaminar el suelo y las aguas y causar epidemias en centros poblados urbanos y rurales, más aun teniendo en cuenta que Potao es un poblado netamente agrícola, lo que al final puede terminar contaminando los productos agrícolas que posteriormente son enviados a la venta al resto de la población. En cuanto a la disposición de basura, el $83.7 \%$ de las familias amontonan la basura en la calle o la arrojan a la acequia, esto es un factor de riesgo debido a que al dejar la basura expuesta se propagan las moscas, lo que permitiría la repetición del ciclo para las infecciones parasitarias.

\section{CONCLUSIONES}

La prevalencia de parasitosis en la población infantil menor de 14 años fue alta, con $71,8 \%$, debido a que en la comunidad de Potao, existe un escaso saneamiento ambiental. El piso de tierra, fue altamente significativo para la presencia de parasitosis, debido a que en ella y a través de la materia fecal se depositan los huevos de parásitos. 
La mala eliminación de las excretas, es altamente significativa para la infestación por parásitos, debido a que pueden contaminar el suelo y el agua, más aún teniendo en cuenta que Potao es un poblado netamente agrícola.

La población infantil de Potao, ticne en su mayoría infestación por Giardia lamblia, Enterobius vermicularis y Escherichia coli.

La Inayoría de lus niños están infestados con al menos un tipo de parásito intestinal $(58.8 \%)$, seguido por los que tuvieron más de dos tipos de parásitos $(41.2 \%)$.

No se encontró asociación entre parasitosis intestinal y la desnutrición de los niños, encontrando que los niños con parasitosis intestinal tienen un estado nutricional normal en un $67 \%$ en comparación con un $33 \%$ de niños con algún grado de malnutrición.

La desnutrición crónica fue encontrada en ambos grupos, entre un $16.7 \%$ a $17 \%$, lo que denota las malas condiciones socioeconómicas, de saneamiento e infecciones parasitarias.

\section{AGRADECIMIENTO}

A la comunidad de Potao-Barranca, por su apoyo incondicional en la información para el estudio. A nuestros Maestros de Post Grado de la Universidad San Pedro de Chimbote, que apoyaron con sus críticas constructivas a este tipo de trabajos.

\section{REFERENCIAS BIBLIOGRÁFICAS}

A. Arancón, J. Segura, I. Galán, J. Trapero, J. Maqueda. (1990). Estudio de portadores familiares en 132 casos parasitados por Giardia lamblia. Aten Primaria; 7 (6): 416-418

N. Batista, M. López de Lama, S. Muñoz, J. Fernández, M. Merino, J. Duque. (1992). Prevalencia de enteropatógenos en guarderías urbanas. Rev San Hig Pública: 66 (5/6): 291-298.

S. Booker, N. Peshu, P. Warn, M. Mosobo, H. Guyatt, K. Marsh. (1999). The epidemiology of hookworm infection and its contribution to anemia among pre-shool children on the Kenyan coast. Trans R Soc. Trop Med Hyg.93:240-46.

D. Botero, M. Restrepo. (1998). Parasitosis Humana. 3ra Edición. Medellín.: Corporación para investigaciones Biológicas.

I. Díaz, L. Botero, F. Ledesma. (2000). Prevalencia de enteroparasitosis en individuos que acuden a la Unidad Docente Asistencial de Medicina Familiar "Luis Sergio Pérez". Kasmera; 28: 45-62.

Instituto Nacional de Estadística e Informática. INEI. (2011). Perú. Indicadores de los Programas Estratégicos, 2010. Lima.

P. Mendoza De Gives, C. Rodríguez, D. Sergio, C. Rudríguez. (2006). Epidemiolugía de las enfermedades parasitarias en animales domésticos. Departamento de parasitología de la Facultad de Medicina Veterinaria. Mèxico.

C. Martínez. (2011). Estadística y muestreo. Eco. Editores. Bogotá. Colombia.

M. Raymundo, V. Terashima, A. Samalvides, y E. Gotuzzo. (2002). Prevalencia de parasitosis intestinal en niños del valle del Mantaro, Jauja, Perú. Rev. Medica Herediana. v. 13 n.3.

URL:http://www.scielo.org.pe/scielo.php?script=sci arttext\&pid=S1018130X2002000300003

\section{Correspondencia}

Juan De La Rosa Díaz Ortiz. Calle 28 de Julio $N^{\circ} 1132$. Cajamarca - Perú. j.díazortiz@yahoo.com.mx

58 Rev. Pakamuros 1 (2): 53-58, 2013 ISSN: 2306-9805 